\title{
Dietary polyphenols and their effect on the gut microbiota and human health
}

\author{
Katja Bezek, Darja Barlič - Maganja \\ University of Primorska, Faculty of Health Sciences, Polje 42, 6300 Izola, Slovenia \\ katja.bezek@fvz.upr.si, darja.maganja@fvz.upr.si
}

\begin{abstract}
Problem presentation: Over the past decade the oxidative stress, caused by reactive oxygen species (ROS) has been recognized as a key factor in the development of various diseases e.g. diabetes, cardiovascular diseases and neurodegenerative disorders. Antioxidant support, which can also be provided with proper nutrition, can reduce the negative effects of oxidative stress and have a positive effect on our health. Plant foods are a rich source of biologically active compounds, among them many polyphenols are very important. Due to their well-known antioxidant properties, polyphenols are associated with a number of physiological mechanisms that have protective effects on various organs, including the gastrointestinal tract. Polyphenols and their metabolites help to maintain a healthy gut primarily through microbiota modulation. They have prebiotic-like effects, they can stimulate the growth of beneficial and inhibit the growth of pathogenic bacteria. In addition, the gut microbiota plays an important role in the metabolism of polyphenols, the production of active metabolites and their bioavailability. Therefore, the interaction between dietary polyphenols and gut microbiota can be of significant benefit to human health. Purpose: The aim of this review is to summarize the data on the protective role of dietary polyphenols and their metabolites on human health in general with an emphasis on gut microbiota modulation. Conclusions: Current research indicates that there is a positive relationship between dietary polyphenols and the healthy composition of the gut microbiota. Therefore, the promotion of a diet rich in plant foods should also be considered as an important element affecting the health of the working population.
\end{abstract}

Keywords: dietary polyphenols, gut microbiota, human health 


\section{Introduction}

Dietary polyphenols represent a large class of naturally occurring chemical compounds characterized by the presence of multiple phenol structural units. As secondary metabolites, they are found widely in plant foods providing colour, flavour and astringency, and defence against exogenous stresses, like reactive oxygen species (ROS), ultraviolet radiation (UV) and plant pathogens. Due to the electron-donating phenolic groups polyphenols are well known antioxidants that prevent stress-related cellular and extracellular damage. In humans, they have been found to possess important biological activities, including anti-inflammatory, anticarcinogenic and antimicrobial activities (Zhang, 2015). Several epidemiologic studies have shown that consumption of food rich in polyphenols has beneficial effects on human health. Their antioxidant and anti-inflammatory properties have preventive effects on different chronic diseases such as cardiovascular diseases, diabetes, obesity, neurodegenerative disorders and cancer (Fraga et al., 2019; Li et al., 2014). Most polyphenols pass through the small intestine without being absorbed, thus encountering the gut microbiota (Ozdal et al., 2016). This has led to the development of a two-way mutual relationship between polyphenolic compounds and gut microbiota. First, polyphenols are biotransformed by gut microbiota that results in the increased bioavailability of their metabolites. The microbiota is responsible for the extensive breakdown of the original polyphenolic structures into low-molecular-weight phenolic metabolites that can be easily absorbed and may actually be responsible for the health effects derived from polyphenol-rich food consumption (Cardona et al., 2013). Second, polyphenols modulate the composition of the gut microbial community mostly through the inhibition of pathogenic bacteria and the stimulation of beneficial bacteria. The last is supported by their prebiotic properties enriching the beneficial bacteria (Valdés et al., 2015). Therefore, the interactions of dietary polyphenols and gut microbiota may impact human health.

\section{Polyphenols characterization and bioavailability}

Polyphenols are classified into a range of structurally related groups, with over 9000 different structures identified in various plant species. This heterogeneous group of molecules, divided into four main classes according to their chemical structure: flavonoids (including flavonols, flavanols, flavanones, flavones, anthocyanidins, chalcones, dihydrochalcones, dihydroflavonols and isoflavones), lignans, stilbenes and tannins. Phenolic acids (hydroxybenzoic, hydroxycinnamic, hydroxyphenylacetic, hydroxyphenylpropanoic and hydroxyphenylactic acids) are also frequently included in this category (Abbas et al., 2017).

Most dietary polyphenols exists as polymers or in glycosylated forms, in which one or more sugar moieties are bound to phenolic or a hydroxyl group at the C-3 position (Manach et al., 2004). The basic structure of flavonoids, meaning the structure of the aglycon form, and which type of sugar moiety is at- 
tached strongly affect their bioavailability. Bioavailability is a crucial factor in determining their biological activity in vivo (Manach et al., 2005).

The bioavailability of dietary polyphenols is, in general, low. Small amounts of their intake (about 5-10 \%) may be absorbed in the small intestine, mainly those with monomeric and dimeric structures. The released aglycones enter the enterocytes by passive diffusion. Once absorbed, polyphenols reach the liver through the portal circulation. Here, they undergo biotransformation via phase I (oxidation, reduction and hydrolysis) and phase II (conjugation) reactions. These transformations produce water-soluble conjugated metabolites (glucuronide, sulphate and methyl derivatives) which are released in the systemic circulation for subsequent delivery to organs and excretion by the urine. More complex polyphenols, especially oligomeric, and polymeric structures, reach the colon almost unchanged, where they are metabolized by the gut $\mathrm{mi}-$ crobiota together with conjugates excreted into the intestinal lumen through the bile. Here, they undergo microbial enzyme transformations, including C-ring cleavage, decarboxylation, dehydroxylation, and demethylation. The result is the generation of less complex compounds such as phenolic acids and hydroxycinnamates (Corrêa et al., 2019).

\section{Polyphenols and gut microbiota modulation}

The human gut is an ecosystem of a round $10^{13}-10^{14}$ bacterial cells, participating in several metabolic functions that the host cannot fulfil by itself. Microbiota that colonize the distal regions of the colon represent the highest concentration of microorganisms found in human body, as well as the most diverse. A harmonious balance in their composition has been associated with maintaining health and a higher life expectancy accompanied by a satisfactory quality of life (Nicholson et al., 2012). The mechanisms by which the phenolic compounds modulate the gut microbiota still remain to be elucidated, but may involve direct and indirect interactions. Phenolic compounds could directly stimulate or inhibit bacterial growth. Inhibition is closely related to the antimicrobial properties of these compounds and stimulation presumably associated with the capacity of the bacteria to metabolize them (Etxeberria et al., 2013). It could be said that polyphenols possess a selective bacteriostatic or bactericidal effect, inhibiting the growth of a wide range of potentially pathogenic bacteria and slightly affecting or even promoting the beneficial microbial population.

Some microbiota members are preferred to others due to efficacy they have shown in ameliorating the gut ecosystem with positive effects at the local and systemic levels. For this reason, most studies have focused on the effects of polyphenols on Bifidobacterium and Lactobacillus, which have been observed to contribute to human health at different levels (Gibson, 2008). They enhance gut barrier function, stimulate the host immune system, prevent diarrhoea or allergies, contribute to activation of provitamins, and modulate lipid metabolism (Burcelin et al., 2012; Gibson, 2008). However, there are other bacterial species associated with negative implications, such as Clostridium dif- 
ficile, which has been associated with inflammatory bowel disease (Rastall et al., 2005). Therefore, it is of crucial importance to understand the inhibitory or stimulatory effect of phenolic compounds on beneficial or pathogenic bacteria. The influence of phenolic compounds on gut microbiota is summarized in details by Ozdal et al. (2016).

In vitro cell culture studies were performed by different polyphenol type substances. Among flavonols tested on six bacteria species (Bacteroides galacturonicus, Lactobacillus spp., Enterococcus caccae, Bifidobacterium catenulatum, Ruminococcus gauvreauii, and Escherichia coli) quercetin showed a dose-dependent inhibitory effect on the growth of all analysed bacterial species, whereas this effect was weaker for rutin (Duda-Chodak, 2012). In another study quercetin supplementation resulted in an altered composition of gut microbiota at different taxonomic levels, including the relative Firmicutes:Bacteroidetes ratio and inhibiting the growth of bacterial species associated with diet-induced obesity such as Erysipelotrichaceae, Bacillus spp., and Eubacterium cylindroides (Etxeberria et al., 2015).

Many different polyphenols were demonstrated to influence the growth of human gut bacteria and their adhesion to enterocytes. Accordingly, naringenin promoted the growth of Lactobacillus rhamnosus, commensal E. coli, along with inhibition of two pathogens, Staphylococcus aureus and Salmonella Typhimurium. In general, the Gram-positive enteropathogen S. aureus was the most sensitive to naringenin, while the Gram-negative pathogen $S$. Typhimurium and the commensal bacteria $E$. coli were likely to be similar in their sensitivity to naringenin (Parkar et al., 2008). The impact of naringenin and hesperetin was tested on six bacteria species (Bacteroides galacturonicus, Lactobacillus sp., E. caccae, B. catenulatum, R. gauvreauii, and E. coli) and inhibited the growth of almost all analysed bacteria (Duda-Chodak, 2012).

Isoflavones are transformed by gut microbiota, although there are few studies regarding the effect of isoflavone supplementation on gut microbiota composition. The investigated isoflavones (e.g. daidzein and genistein) induced a decrease in bacterial growth (Kawabata et al., 2013). The consumption of flavanol-rich foods containing epicatechin and catechin may support gut health through their ability to exert prebiotic actions (Tzounis et al., 2008). The flavan-3-ol modulates microbiota composition and inherent catabolic activity, inducing changes that could affect the bioavailability and potential bioactivity of these compounds (Cueva et al., 2013).

Anthocyanins and their metabolites may stimulate beneficial members of the gut microta community. Interestingly, malvidin-3-glucoside mixed with other anthocyanins exhibited a synergistic effect in promoting beneficial microbes. In vitro incubation of phenolic gallic acid in a fecal slurry reduced a group of potentially harmful bacteria such as Clostridium histolyticum without any negative effects on beneficial bacteria. In addition, it significantly reduced Bacteroides spp. growth and enhanced both the total bacterial number and the abundance of Atopobium spp. (Hidalgo et al., 2012). In another 
study, the influence of hydroxycinnamic acids such as caffeic acid, chlorogenic acid, o-coumaric acid, p-coumaric acid on the growth of a probiotic microbe (L. rhamnosus), a commensal (E. coli) and two pathogenic bacteria ( $S$. aureus, $S$. Typhimurium) was investigated. They compared the MIC values of all polyphenols tested and observed that flavonols, isoflavones and glycosides have low antibacterial activity, while phenolic acids were found to be at an intermediate level. On the other hand, the flavanone and flavanol had high antibacterial activity (Parkar et al., 2008).

The effect of hydrolysable tannins (ellagitannins) on the growth of intestinal bacteria is inadequately characterized, and generally their antimicrobial potential has been assessed in vitro. It was observed that pomegranate by-products and punicalagins inhibited the growth of pathogenic Clostridia and S. aureus. Interestingly, probiotic lactobacilli and bifidobacteria were generally not affected by ellagitannins (Bialonska et al., 2009). In this experiment, pomegranate extract was able to increase the total bacterial number, enhancing the growth of Bifidobacterium spp., Lactobacillus and Enterococcus groups, while no effect was observed for the C. histolyticum group (Bialonska et al., 2010).

Resveratrol, the representative of stilbenes, increased lactobacilli and bifidobacteria as well as diminished the increase of enterobacteria in in vivo studies (Larrosa et al., 2009) not representative from a dietary point of view. Our aim was to ascertain whether resveratrol can exert anti-inflammatory activity in vivo at an attainable dietary dose. Rats were fed with $1 \mathrm{mg}$ of resveratrol/ $\mathrm{kg} /$ day (a human equivalent dose). The results of another study showed that resveratrol ameliorates the dysbiosis in the gut microbiota induced by the highfat diet, specific effects include an increase in the Bacteroidete:Firmicutes ratio, significant inhibition of the growth of Enterococcus faecalis, and increased growth of Lactobacillus and Bifidobacterium (Qiao et al., 2014).

The main limitation of the presented studies is that the information obtained from in vitro studies about the role of individual phenolic compounds on gut microbiota cannot be directly extrapolated to what occurs in the physiological context of the gut ecosystem. Of those performed, most were focused on a single polyphenol molecule and selected bacterial populations. Most phenolic fractions and pure phenolic compounds have been analysed without considering the bioavailability and the chemistry of phenolic compounds in the colon. Human and animal intervention studies involved very high doses of individual phenolic compound, or high amounts of foods rich in phenolic content, neither of which represents the regular diet (Ozdal et al., 2016). Therefore, there is a lack of adequate in vivo studies which are needed to understand the effect of phenolic compounds on gut microbiota. Human intervention studies will provide the best models for studying the effect of phenolic compounds on gut microbiota modulation. There may be a highly variable response to phenolic compounds according to the differences in gut microbiota composition. Future studies should provide answers about the inter-individual differences in 
gut microbiota while studying the effect of phenolic compounds on gut microbiota modulation from the immunological point of view.

\section{Conclusions}

Dietary polyphenols have increasingly interested the scientific community due to their proposed health benefits. Possible beneficial effects of polyphenols are determined by their bioavailability where the gut microbiota have an important role. Phenolic compounds are biotransformed into their smaller metabolites by gut microbiota, which contributes to increased bioavailability. At the same time, phenolic compounds can alter the gut microbiota community, resulting in a greater abundance of beneficial microbes, and a consequent increase in bioavailability. Despite the results provided and published over recent years, future studies including human trials will give more confirmatory results about the efficacy of phenolic compounds at the gut level. These results may lead to the design of dietary recommendations not only to suppress or reduce symptoms in disease but also to provide the healthy population with simple tools to promote the maintenance of health.

\section{References}

Abbas, M., Saeed, F., Anjum, F. M., Afzaal, M., Tufail, T., Bashir, M. S., Ishtiaq, A., Hussain, S., \& Suleria, H. A. R. (2017). Natural polyphenols: An overview. International Journal of Food Properties, 2o(8), 1689-1699. https:// doi.org/10.1080/10942912.2016.1220393

Bialonska, D., Kasimsetty, S. G., Schrader, K. K., \& Ferreira, D. (2009). The effect of pomegranate (Punica granatum L.) byproducts and ellagitannins on the growth of human gut bacteria. Journal of Agricultural and Food Chemistry, 57(18), 8344-8349. https://doi.org/10.1021/jf901931b

Bialonska, D., Ramnani, P., Kasimsetty, S. G., Muntha, K. R., Gibson, G. R., \& Ferreira, D. (2010). The influence of pomegranate by-product and punicalagins on selected groups of human intestinal microbiota. International Journal of Food Microbiology, 140(2), 175-182. https://doi.org/10.1016/j.ijfoodmicro.2010.03.038

Burcelin, R., Garidou, L., \& Pomié, C. (2012). Immuno-microbiota cross and talk: The new paradigm of metabolic diseases. Seminars in Immunology, 24(1), 67-74. https://doi.org/10.1016/j.smim.2011.11.011

Cardona, F., Andrés-Lacueva, C., Tulipani, S., Tinahones, F. J., \& Queipo-Ortuño, M. I. (2013). Benefits of polyphenols on gut microbiota and implications in human health. The Journal of Nutritional Biochemistry, 24(8), 1415-1422. https://doi.org/10.1016/j.jnutbio.2013.05.001

Corrêa, T. A. F., Rogero, M. M., Hassimotto, N. M. A., \& Lajolo, F. M. (2019). The Two-Way Polyphenols-Microbiota Interactions and Their Effects on 
Obesity and Related Metabolic Diseases. Frontiers in Nutrition, 6. https:// doi.org/10.3389/fnut.2019.00188

Cueva, C., Sánchez-Patán, F., Monagas, M., Walton, G. E., Gibson, G. R., Martín-Álvarez, P. J., Bartolomé, B., \& Moreno-Arribas, M. V. (2013). In vitro fermentation of grape seed flavan-3-ol fractions by human faecal microbiota: Changes in microbial groups and phenolic metabolites. FEMS Microbiology Ecology, 83(3), 792-805. https://doi.org/10.1111/15746941.12037

Duda-Chodak, A. (2012). The inhibitory effect of polyphenols on human gut microbiota. Journal of Physiology and Pharmacology: An Official Journal of the Polish Physiological Society, 63(5), 497-503.

Etxeberria, U., Arias, N., Boqué, N., Macarulla, M. T., Portillo, M. P., Martínez, J. A., \& Milagro, F. I. (2015). Reshaping faecal gut microbiota composition by the intake of trans-resveratrol and quercetin in high-fat sucrose diet-fed rats. The Journal of Nutritional Biochemistry, 26(6), 651-66o. https://doi.org/10.1016/j.jnutbio.2015.01.002

Etxeberria, Usune, Fernández-Quintela, A., Milagro, F. I., Aguirre, L., Martínez, J. A., \& Portillo, M. P. (2013). Impact of Polyphenols and Polyphenol-Rich Dietary Sources on Gut Microbiota Composition. Journal of Agricultural and Food Chemistry, 61(40), 9517-9533. https://doi.org/10.1021/ jf $402506 \mathrm{c}$

Fraga, C. G., Croft, K. D., Kennedy, D. O., \& Tomás-Barberán, F. A. (2019). The effects of polyphenols and other bioactives on human health. Food \& Function, 10(2), 514-528. https://doi.org/10.1039/C8FO01997E

Gibson, G. R. (2008). Prebiotics as gut microflora management tools. Journal of Clinical Gastroenterology, 42 Suppl 2, S75-79. https://doi.org/10.1097/MCG. obo13e31815edo97

Hidalgo, M., Oruna-Concha, M. J., Kolida, S., Walton, G. E., Kallithraka, S., Spencer, J. P. E., Gibson, G. R., \& de Pascual-Teresa, S. (2012). Metabolism of Anthocyanins by Human Gut Microflora and Their Influence on Gut Bacterial Growth. Journal of Agricultural and Food Chemistry, 6o(15), 3882-389o. https://doi.org/10.1021/jf3002153

Kawabata, K., Sugiyama, Y., Sakano, T., \& Ohigashi, H. (2013). Flavonols enhanced production of anti-inflammatory substance(s) by Bifidobacterium adolescentis: Prebiotic actions of galangin, quercetin, and fisetin. $\mathrm{Bi}$ oFactors, 39(4), 422-429. https://doi.org/10.1002/biof.1081

Larrosa, M., Yañéz-Gascón, M. J., Selma, M. V., González-Sarrías, A., Toti, S., Cerón, J. J., Tomás-Barberán, F., Dolara, P., \& Espín, J. C. (2009). Effect of a Low Dose of Dietary Resveratrol on Colon Microbiota, Inflammation and Tissue Damage in a DSS-Induced Colitis Rat Model. Journal of Agricultural and Food Chemistry, 57(6), 2211-2220. https://doi.org/10.1021/ jf8o3638d 
Li, A.-N., Li, S., Zhang, Y.-J., Xu, X.-R., Chen, Y.-M., \& Li, H.-B. (2014). Resources and Biological Activities of Natural Polyphenols. Nutrients, 6(12), 6020-6047. https://doi.org/10.339o/nu6126020

Manach, C., Scalbert, A., Morand, C., Rémésy, C., \& Jiménez, L. (2004). Polyphenols: Food sources and bioavailability. The American Journal of Clinical Nutrition, 79(5), 727-747. https://doi.org/10.1093/ajcn/79.5.727

Manach, C., Williamson, G., Morand, C., Scalbert, A., \& Rémésy, C. (2005). Bioavailability and bioefficacy of polyphenols in humans. I. Review of 97 bioavailability studies. The American Journal of Clinical Nutrition, 81(1), 230S-242S. https://doi.org/10.1093/ajcn/81.1.230S

Nicholson, J. K., Holmes, E., Kinross, J., Burcelin, R., Gibson, G., Jia, W., \& Pettersson, S. (2012). Host-Gut Microbiota Metabolic Interactions. Science, 336(6086), 1262-1267. https://doi.org/10.1126/science.1223813

Ozdal, T., Sela, D. A., Xiao, J., Boyacioglu, D., Chen, F., \& Capanoglu, E. (2016). The Reciprocal Interactions between Polyphenols and Gut Microbiota and Effects on Bioaccessibility. Nutrients, 8(2), 78. https://doi.org/10.339o/ nu 8020078

Parkar, S. G., Stevenson, D. E., \& Skinner, M. A. (2008). The potential influence of fruit polyphenols on colonic microflora and human gut health. International Journal of Food Microbiology, 124(3), 295-298. https://doi. org/10.1016/j.ijfoodmicro.2008.03.017

Qiao, Y., Sun, J., Xia, S., Tang, X., Shi, Y., \& Le, G. (2014). Effects of resveratrol on gut microbiota and fat storage in a mouse model with high-fat-induced obesity. Food \& Function, 5(6), 1241-1249. https://doi.org/10.1039/ c3fo6o63oa

Rastall, R. A., Gibson, G. R., Gill, H. S., Guarner, F., Klaenhammer, T. R., Pot, B., Reid, G., Rowland, I. R., \& Sanders, M. E. (2005). Modulation of the microbial ecology of the human colon by probiotics, prebiotics and synbiotics to enhance human health: An overview of enabling science and potential applications. FEMS Microbiology Ecology, 52(2), 145-152. https:// doi.org/10.1016/j.femsec.2005.01.003

Tzounis, X., Vulevic, J., Kuhnle, G. G. C., George, T., Leonczak, J., Gibson, G. R., Kwik-Uribe, C., \& Spencer, J. P. E. (2008). Flavanol monomer-induced changes to the human faecal microflora. The British Journal of Nutrition, 99(4), 782-792. https://doi.org/10.1017/Sooo7114507853384

Valdés, L., Cuervo, A., Salazar, N., Ruas-Madiedo, P., Gueimonde, M., \& González, S. (2015). The relationship between phenolic compounds from diet and microbiota: Impact on human health. Food \& Function, 6(8), 2424-2439. https://doi.org/10.1039/C5FOoo322A

Zhang, P.-Y. (2015). Polyphenols in Health and Disease. Cell Biochemistry and Biophysics, 73(3), 649-664. https://doi.org/10.1007/s12013-015-0558-Z 